\title{
Aggregatibacter (Actinobacillus) Actinomycetemcomitans y Enfermedad Periodontal.
}

Periodontal disease and Aggregatibacter (Actinobacillus) actinomycetemcomitans.

Fecha de Recepción

I8 de marzo de 201 I
Aggregatibacter (Actinobacillus) actinomy-

cetemcomitans e doença periodontal.

Aceptado para su publicación

| 3 de abril de 20 | |
Silvia Ortega

Profesor Adjunto a cargo Cátedra de Microbiología e Inmunología. Facultad de Odontología.

Olga Vasek

Profesor Adjunto Cátedra de Biotecnología. Facultad Ciencias Exactas, Naturales y Agrimensura.

\section{Cynthya Sin}

Becaria de Pregrado de Ciencia y Técnica. UNNE.

Lugar de trabajo Facultad de Odontología. UNNE. Av. Libertad 5450 - 3400 Corrientes Capital Argentina. Tel/fax: 03783457992.

E-mail: silviaortega I4@yahoo.com.ar omvasekk@yahoo.com.ar cynthya_cysin2I@hotmail.com

\section{Resumen}

La enfermedad periodontal ha sido asociada siempre al biofilm o placa dental. El mismo está compuesto por microorganismos taxonómicamente diversos y se localiza principalmente, en el surco gingival. Su composición es tan compleja, que la mayoría de las bacterias comensales de la cavidad bucal están presentes. En el biofilm que produce enfermedades gingivoperiodontales, las bacterias Gram negativas representan la biota dominante $y$ entre ellos Aggregatibacter (Actinobacillus) actinomycetemcomitans reviste particular importancia. Este microorganismo posee elementos estructurales y genera distintos productos que se comportan como antígenos frente al huésped, quien responde de maneras diferentes, siendo éste un factor esencial en la progresión de la enfermedad. Es importante conocer la patogenicidad de la población constituyente del biofilm y su acción sobre los tejidos periodontales, así como, destacar que el buen diagnóstico tanto clínico como radiográfico sumado al estudio microbiológico permitirá instaurar el tratamiento adecuado para el mejor pronóstico evitando eventuales complicaciones sistémicas que pudieran comprometer el estado general del paciente.

\section{Palabras claves}

Microorganismos periodontopatógenos, diagnóstico microbiológico, diagnóstico precoz en periodontitis. 


\section{Abstract}

Periodontal disease has always been associated biofilm or plaque. The same is composed of several different organisms and is found especially in the sulcus. Its composition is so complex that most of commensal bacteria in the oral cavity are present. In the disease-causing biofilm gingivoperiodontales Gram-negative bacteria represent the dominant biota including Aggregatibacter (Actinobacillus) actinomycetemcomitans is particularly important. This organism possesses structural elements and generates various products that act as antigens from the host, who responds in different ways, and this is an essential factor in the progression of the disease. It is important to understand the pathogenicity of the population constituent of biofilm and its effect on periodontal tissues, as well as highlight the good clinical and radiographic diagnosis coupled with microbiological study will establish the appropriate treatment for the best prognosis, avoiding potential systemic complications could compromise the patient's general condition.

\section{Keywords}

Periodontal microorganisms, microbiological diagnosis, early diagnosis in periodontitis.

\section{Resumo}

Sido sempre associado biofilme dental ou placa bacteriana. É composta por microrganismos taxonomicamente diversas e situam-se principalmente no sulco. Sua composição é tão complexo que a maioria das bactérias comensais na cavidade oral estão presentes. No gingivoperiodontales biofilme causadores de doenças bactérias Gram-negativas representam a biota dominante incluindo Aggregatibacter (Actinobacillus) actinomycetemcomitans é particularmente importante. Este organismo possui elementos estruturais e gera produtos que atuam como antígenos do hospedeiro, que responde de maneiras diferentes, e este é um factor essencial para a progressão da doença. É importante entender a patogenicidade do componente de população de biofilme e seu efeito sobre os tecidos periodontais, bem como destacar o bom diagnóstico clínico e radiográfico juntamente com estudo microbiológico irá estabelecer $\circ$ tratamento adequado para $\circ$ melhor prognóstico, evitando possíveis complicações sistêmicas poderia comprometer o estado geral do paciente.

\section{Palavras chaves \\ Microrganismos periodontais, diagnóstico microbiológico, o diagnóstico precoce de periodontite.}

\section{Introducción}

Las enfermedades periodontales son procesos inflamatorios que afectan a los tejidos de protección y sostén de las piezas dentarias. La inflamación de las encías o gingivitis se relaciona con la placa supragingival, las periodontitis, con la placa subgingival e involucran la destrucción de los tejidos periodontales de sostén de la pieza dentaria y se relacionan.

Las periodontitis poseen una etiopatogenia compleja que puede considerarse multifactorial, ya que además de la presencia de biofilm, otros factores relacionados a la respuesta del huésped' se asocian a ella.

El biofilm, entendido como una entidad dinámica, agrupa bacterias en un material amorfo de triple origen con actividad metabólica propia y varía según su nicho ecológico o ecosistema primario. Un gran número de bacterias Gram positivas están relacionadas con estados de salud; cambios en el microambiente, favorecen el aumento de bacterias Gram negativas anaerobias facultativas $y$ anaerobias estrictas, usualmente asociadas a enfermedad periodontal. Una de las características del biofilm dental es que las bacterias constituyentes desarrollan frecuentemente fenotipos diferentes a los de las bacterias planctónicas, situación por la cual algunas exhiben mayor tolerancia a los antibióticos y otros parámetros ambientales como $\mathrm{pH}$ y oxígeno?

La placa subgingival se localiza en el surco gingival y va formándose por colonización, agregación y co-agregación bacteriana. En estado de salud existe, en el surco gingival, una microbiota normal compatible con el huésped. Un aumento tanto en cantidad como en diversidad dará inicio a la periodontitis, a la que el huésped responderá ${ }^{3}$ en forma normal o patológica, y que a su vez será específica o no. Una respuesta inmune disminuida por parte del huésped, estará relacionada a factores de riesgo que aumentarán las proba- 
bilidades de desarrollo de la enfermedad. Estos factores pueden ser de tipo genético, ambientales tales como estrés, edad, higiene deficiente, consumo de tabaco, condición socio-económica, obesidad ${ }^{4}$.

Desde el punto de vista microbiológico, Haffajee y Socransky ${ }^{5}$ sugirieron el agrupamiento de los microorganismos que se hospedan en el surco gingival. Un grupo compuesto por Streptococcus (S.) mitis, S. oralis, S. sanguinis, S. intermedius, $S$. gordonii, Actinomyces naeslundii y odontoliticus, $y$ Veilonella spp., un grupo formado por Eikenella corrodens, Capnocitophaga spp. y Aggregatibacter actinomycetemcomitans (A.a) serotipo a $y$, al grupo que incluye a Fusobacterium nucleatum y periodonticum. Estos últimos constituyen un nexo con bacterias de aparición tardía ${ }^{6}$, tales como Prevotella intermedia y nigrescens y Peptoestreptococcus micros. Posteriormente, se adicionarán Porphyromona gingivalis, Tannerella forsythia y Treponema dentícola, microorganismos relacionados con el desarrollo y progreso de la enfermedad periodontal que han sido denominados como grupo rojo.

La periodontitis crónica aparece luego de los 35 años y progresa lentamente, presentando episodios de agudización y luego remisión. La periodontitis agresiva se caracteriza por una pérdida de inserción y destrucción ósea rápida.

Además de los microorganismos componentes del biofilm, la respuesta del huésped es otro factor a considerar en el desarrollo de estas lesiones. Varias son las investigaciones que se han realizado para establecer la respuesta del huésped, entre ellas la determinación de inmunoglobulinas tanto séricas como las presentes en el fluido crevicular. Actualmente los avances tecnológicos permiten determinar la presencia de distintos productos o metabolitos inflamatorios de la respuesta celular y humoral del huésped. Estos biomarcadores, de los cuales algunos indican destrucción tisular, permiten detectar precozmente pacientes de riesgo y así instaurar el tratamiento adecuado en forma temprana. En el fluido crevicular puede determinarse, también, la presencia de células polimorfonucleares y mediadores séricos como factores del complemento, del sistema linfático y quininas.

Por lo tanto es necesario conocer la etiopatogenia de la enfermedad, la virulencia de las bacterias asociadas, su sensibilidad a los antibióticos así como el estado general del huésped y su res- puesta, a fin de lograr un tratamiento que garantice el pronóstico de la enfermedad periodontal.

\section{Desarrollo}

Entre los microorganismos reconocidos como periodontopatógenos se encuentra Aggregatibacter (Actinobacillus) actinomycetemcomitans (A.a); es un cocobacilo o bacilo pequeño Gram negativo (figura I), inmóvil, anaerobio facultativo o capnofilico y sacarolítico. Es reconocido como habitante normal de la mucosa oral, de la placa dental y los surcos gingivales pudiendo ocasionar infecciones en otras partes del cuerpo por diseminación. En 1976 se lo halló con frecuencia asociado a periodontitis juvenil localizada; sin embargo también puede contribuir al desarrollo de otras formas de periodontitis ${ }^{7}$.

Tiene como factores de virulencia elementos estructurales y productos extracelulares. Presenta fimbrias que favorecen la adhesión a células epiteliales de la encía, así como capacidad de autoagregación tanto en el biofilm como en medios de cultivo, en los que las poblaciones microbianas desarrollan formas extremadamente adherentes a la superficie vítrea del recipiente contenedor en laboratorio y a otras estructuras abióticas ${ }^{8}$.

Como organismo Gram negativo posee lipopolisacáridos (LPS) en su pared, que poseen una fuerte acción sobre la estructura celular alterando la organización de las fibras de actina y tubulina que contribuyen al citoesqueleto de fibroblastos humanos?.

Figura I. Coloración de Gram: cocobacilos negativos. 
El polisacárido $O$ que forma dicho LPS permite clasificar a esta bacteria en seis serotipos: $a, b$, $c, d, e, f$, al que se adiciona el $g$, recientemente descubierto ${ }^{10}$. Los serotipos $a, b$ y $c$ son los que se recuperan más frecuentemente de la cavidad oral en comparación a los serotipos $d y$ e, considerándose a los serotipos $b$ y $c$ como los de mayor virulencia"l.

El serotipo más asociado a periodontitis agresiva, antes periodontitis juvenil localizada, es el $b$, que asimismo se asocia con la producción de betalactamasas, bacteremia y endocarditis, y el serotipo $c$ que se aísla principalmente de infecciones extraorales y de cavidades orales sanas ${ }^{12}$. Aggregatibacter actinomycetemcomitans serotipo $b$, se determinó como biota dominante en sujetos con enfermedad periodontal y el serotipo $c$ fue el más habitualmente hallado en sujetos sin enfermedad periodontal' ${ }^{13}$. Sin embargo Chen y colaboradores ${ }^{14}$ asociaron a este último serotipo con periodontitis agresivas generalizadas.

Estudios realizados en diferentes países fueron enfocados a determinar la distribución de los distintos serotipos en poblaciones diversas. Algunos autores detectaron ${ }^{15,16}$ prevalencia del serotipo $c$ en niños finlandeses y vietnamitas. RomanTorres $^{17}$ informó la presencia de anticuerpos contra A.a serotipo $b$, en niños con Síndrome de Down en Brasil, y en grupos con periodontitis leve y moderada los serotipos $c$ y $a$, constituyendo el primero el de mayor relevancia en periodontitis severa, en forma coincidente con reportes previos referidos a poblaciones asiáticas y europeas. En Estados Unidos, se reveló al serotipo c como el más frecuente seguido por $a$ y $b$. Dado que los otros serotipos no fueron detectados, en los patrones de distribución de los serotipos podrían influir la raza y etnia ${ }^{14}$ o factores relacionados al microorganismo en sí mismo como una estrategia de colonización crónica ${ }^{18}$.

Los serotipos pueden determinarse hoy día, mediante técnicas moleculares como PCR, PCR Multiplex, técnicas de inmunofluorescencia $O$ inmunodifusión (ELISA) y otras.

Aggregatibacter actinomycetemcomitans produce dos toxinas, una conocida como toxina citoletal, exclusiva de este microorganismo que ejerce esta actividad sobre células epiteliales. La producción de esta toxina podría resultar de la adaptación del microorganismo a la respuesta del huésped", particularmente en periodontitis crónicas, indu- ciendo la muerte celular al permitir la formación de poros en sus membranas que alteran la permeabilidad, provocando un ensanchamiento progresivo y muerte posterior ${ }^{19}$.

Leucotoxina es la otra toxina extracelular con capacidad para inhibir la actividad de células polimorfonucleares; otras células que pueden verse afectadas son macrófagos y ciertos linfocitos, no así fibroblastos, células epiteliales y endoteliales ${ }^{20}$.

Esta toxina es liberada a través de vesículas de la membrana externa y está codificada en el cromosoma bacteriano ${ }^{21,11}$. El operón Itx, compuesto por cuatro genes, incluye al gen ItxA estructural $y$ los genes ItxB, ItxC y ltxD que son necesarios para la activación y transporte al exterior de la toxina para llegar a las células del huésped.

Este microorganismo posee además enzimas proteolíticas que incluyen endo y exopeptidasas. Las gingipaínas o endopeptidasas de la cisteína, constituyen las enzimas proteolíticas mayoritarias $^{22}$. Se comportan como hemoaglutininas contribuyendo a la adhesión frente a las células epiteliales, facilitada por la presencia de fimbrias, condición indispensable para la colonización del microorganismo.

Como factor de supervivencia adicional, este microorganismo produce bacteriocinas, proteínas extracelulares capaces de inhibir el desarrollo de otros microorganismos en el biofilm. La más recientemente reportada es la actinobacilina ${ }^{19}$ que ejerce efecto inhibidor contra Peptoestreptococcus anaerobius, Streptococcus sanguinis y Actinomyces spp.

\section{Respuesta del huésped}

Estudios de las últimas décadas demuestran que el sistema inmunológico del huésped y su capacidad defensiva frente a los diversos antígenos bacterianos inducen la liberación de componentes inflamatorios que producen destrucción de tejidos y son responsables del progreso de la enfermedad, que puede tornarse más destructiva si algunos factores del huésped favorecen una hiperrespuesta. Antígenos y productos bacterianos inician una respuesta inflamatoria que resulta en la producción de citoquinas, productos de activación del complemento y metaloproteinasas'. Las metaloproteinasas de la matriz son enzimas degradativas de los tejidos, son producidas por 
varias células y difieren en su función. En el tejido gingival y periodontal se encuentran las degradantes de colágeno tipo I y III producido por fibroblastos siendo su destrucción el rasgo fundamental de la progresión y actividad de periodontitis. Sus niveles en el líquido crevicular son elevados en sitios enfermos al compararse con sitios periodontales sanos. Las relacionadas con periodontitis son la MMP-8 o colagenasa 2 y la MMP-9 o gelatinasa $B$. En periodontitis no tratadas $y$ en formas activas de enfermedad periodontal, se cuantifican elevados niveles de colagenasas ${ }^{23}$, de modo tal que estas sustancias, pueden considerarse como biomarcadores de la destrucción de tejidos, utilizando muestras de saliva, fluido crevicular o suero ${ }^{24}$. También pueden considerarse biomarcadores a las linfoquinas activadoras de osteoclastos (interleuquina I $\beta$ ) y linfotoxinas.

A.a., además de estimular una respuesta inflamatoria inespecífica, es capaz de producir respuesta específica comprobada por la existencia de anticuerpos contra este microorganismo tanto en suero como en líquido crevicular. Su función incluye la habilidad de opsonizar bacterias para que sean removidas por células macrofágicas o fijarse a sus adhesinas como aglutininas, previniendo o minimizando la instalación del microorganismo en el biofilm.

Inmunoglobulina $\mathrm{G}$ en elevados niveles fué determinada en un $90 \%$ de pacientes con periodontitis localizada de aparición temprana, $40 \%$ con periodontitis juvenil localizada y $25 \%$ con periodontitis en adultos 25 , siendo Inmunoglobulina $G$ subclase 2 la que reacciona más frecuentemente contra A. $a^{18}$.

La propuesta actual ${ }^{26}$ consiste en la detección de distintos biomarcadores, entre ellos anticuerpos contra A.a y Porfhyromona gingivalis en saliva, fluido crevicular y suero a fin de facilitar el diagnóstico precoz.

Según Frias Lopez ${ }^{27}$, parece razonable estudiar la respuesta del huésped frente a la agresión y así detectar precozmente la susceptibilidad individual o resistencia a la periodontitis. La aplicación de métodos de diagnóstico basados en el análisis del fluido crevicular brinda información para detectar pacientes en riesgo e implementar el tratamiento adecuado tempranamente.

Aislamiento de A.a a partir de muestras clínicas En 1982, fue propuesto28 un medio de cultivo selectivo para su aislamiento a partir de bolsas periodontales. Este medio (TSVB) contiene tripteína de soja, extracto de levadura enriquecido con suero equino y adicionado de bacitracina y vancomicina. Considerando que dicho medio no reflejaba el recuento real de las bacterias, se diseñó uno nuevo (AASM) que, además de los nutrientes citados anteriormente, adicionaba dextrosa, bicarbonato de sodio y concentraciones superiores de los antibióticos. Esta reformulación, permitió el desarrollo de todos los serotipos del microorganismo eliminando los falsos positivos obtenidos con el medio original ${ }^{29}$. En este nuevo medio como en el otro, en forma agarizada, el microorganismo desarrolla como colonias pequeñas $(0,5-\mathrm{Imm})$, translúcidas o transparentes, (figura 2) con bordes irregulares e inicialmente rugosas debido a la capacidad de autoagregación (figura 3), manteniendo esta característica en medios de cultivo líquidos en los cuales se observa la adhesión poblacional a la pared del tubo contenedor sin turbidez generalizada ${ }^{8}$.

Para evidenciar la presencia de este microorganismo en muestras clínicas, se emplean métodos de inmunofluorescencia indirecta ${ }^{30}$ métodos moleculares, enzimáticos e inmunológicos ${ }^{3}$. Sin embargo, el diagnóstico microbiológico por cultivos es el que permite, además de establecer prevalencia y sensibilidad a diferentes antibióticos, la cuantificación de los mismos, intentando relacionar su diversidad y cuantía con la severidad de la enfermedad.

Algunos autores opinan que las infecciones con A.a. son exógenas debido a que raramente se encuentran en individuos libres de enfermedad periodontal ${ }^{31}$. Otros ${ }^{32}$ sin embargo, consideran que las infecciones periodontales son endógenas, causadas por microorganismos que habitan en la cavidad bucal. La transmisión de A. a. puede ser de tipo horizontal (entre individuos de una misma familia, cónyuges) o de tipo vertical (padres e hijos) aunque la instalación y colonización es multifactorial, dependiendo principalmente del huésped, características de la cepa, número de bacterias inoculadas y tiempo de exposición a la infección ${ }^{33}$.

El diagnóstico de la enfermedad periodontal debe tomar como base la historia clínica, el examen clínico y radiológico, y estudios de laboratorio ${ }^{34}$. Durante el avance de la lesión a episodios tanto 
agudos como crónicos, la interrelación huésped - microorganismos de la placa, atraviesan distintas etapas, de manera tal que el hallazgo de A.a. no necesariamente implica la existencia de una enfermedad periodontal aguda o crónica ${ }^{35,36}$.

Epidemiológicamente, la enfermedad periodontal tiene gran trascendencia. El diagnóstico de este proceso es necesario para proceder a un tratamiento y prevención más adecuada. Actualmente la periodontitis es concebida como una enfermedad que, además de la presencia de biofilm, considera la susceptibilidad del individuo como huésped. Por lo tanto las condiciones ambientales, nivel socioeconómico y hábitos higiénicos pueden incidir en distintos patrones de evolución de la enfermedad. Los brotes o períodos de actividad están relacionados con la presencia de A.a y Porphyromona gingivalis.

El tratamiento de la enfermedad periodontal se basa en terapias de raspaje, curetaje y técnicas quirúrgicas que pueden ir acompañadas de la administración local o sistémica de antibióticos y causan una reducción de la población microbiana, siendo lo ideal que ese porcentaje sea menor al $5 \%$. En un estudio longitudinal en el que se compararon dos grupos, uno con terapia de raspaje y curetaje sin antibióticos y otro con la misma técnica y adición de amoxicilina y metronidazol, se observó que el grupo tratado con antibióticoterapia sistémica disminuyó el riesgo de pérdida de inserción, sin embargo ello no evita la recolonización subgingival o de mucosa oral para los diferentes periodontopatógenos exceptuando a A. Actinomycetemcomitans ${ }^{37}$. Sin embargo otros autores consideran que A.a. resiste a terapias convencionales, por ejemplo tetraciclinas ${ }^{38}$. En otro estudio, se realizó la evaluación de distintos antibióticos administrados por vía oral y se halló que el microorganismo fue altamente susceptible a ciprofloxacina y moxafloxacina no así a azitromicina y metronidazol ${ }^{39}$.

El uso de antibióticos sistémicos junto a una terapia de raspaje y curetaje ha favorecido a los pacientes periodontales en los que se evidenció la presencia de Prophyromona gingivalis y A.a. siendo que estos patógenos no siempre se detectan en sitios enfermos.

Acuña y Hernandez ${ }^{38}$ luego de un meta-análisis referente al uso de antibióticos en el tratamiento de la enfermedad periodontal expresan algunas consideraciones a la hora de su tratamiento.
Figura 2. Colonias obtenidas de un primer aislamiento.

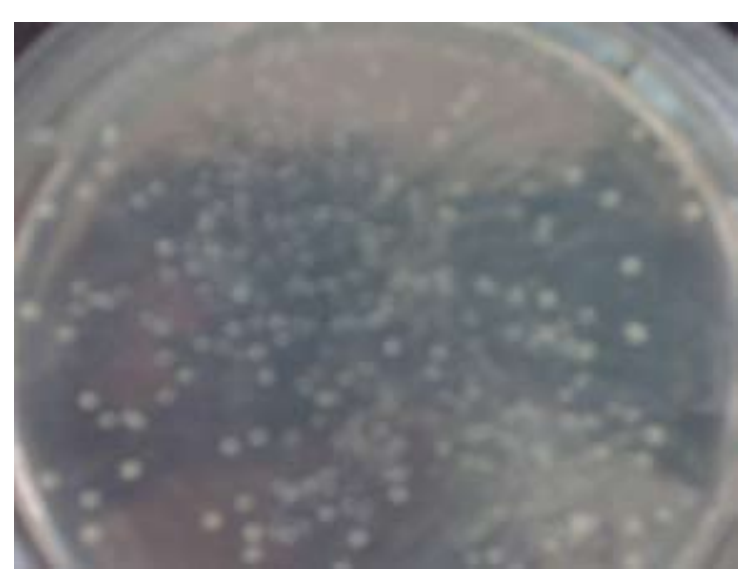

Figura 3. Colonia de Aggregatibacter actynomicetemcomitans vista con lupa estereoscópica.

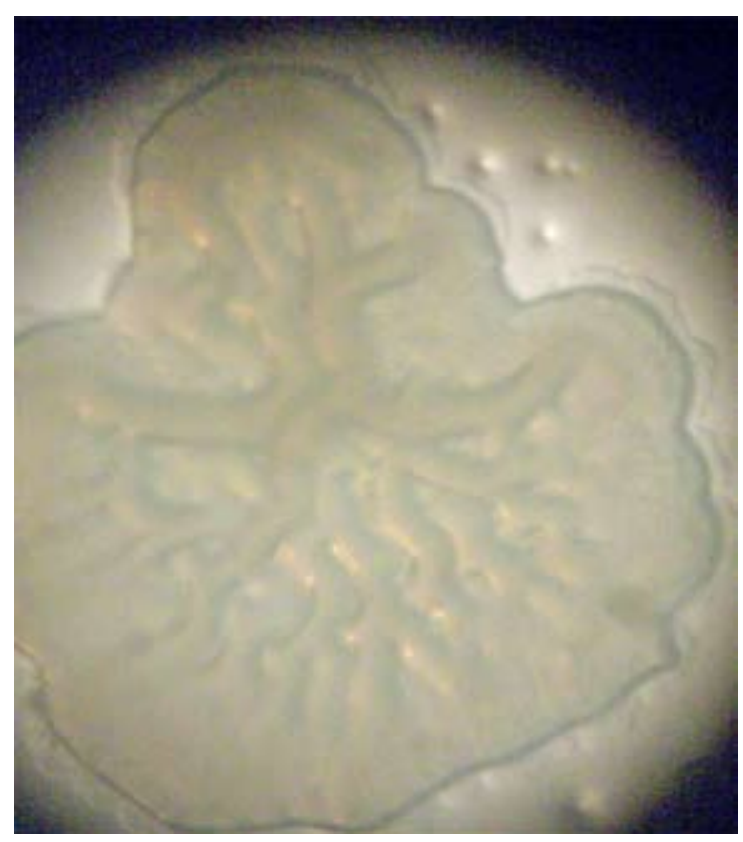

Aconsejan que debe realizarse un diagnóstico clínico periodontal y microbiológico específico para cada paciente al mismo tiempo que la evaluación de la salud general del paciente, la respuesta al tratamiento de alisado y pulido radicular y los posibles efectos adversos de cada antibiótico, prescribiéndolos sólo en la primer fase si los hallazgos microbiológicos confirman la presencia de un patógeno específico así como su sensibilidad, realizando una re-evaluación clínica a los seis meses o entre uno y tres meses; además, al hacer la re-evaluación aconsejan realizar un examen microbiológico y luego de la resolución de la 
infección adicionar una terapia de soporte basada en el control de placa bacteriana.

\section{Conclusión}

Aggregatibacter actinomycetmcomitans es uno de los más importantes microorganismos periodontopatógenos por el rol que desempeña en el inicio y progreso de la enfermedad periodontal. Considerando que se lo encuentra en la placa subgingival, su presencia puede ser prevenida con educación y motivación para mantener la higiene oral, y si la enfermedad ocurre se podrían lograr buenos resultados con tratamiento clásico.

Los biofilms relacionados con gingivitis y periodontitis incluyen una comunidad polimicrobiana compleja, aun cuando existen diferencias en la composición de la microflora subgingival en sujetos de varias regiones geográficas. Es necesario el conocimiento de la composición bacteriana en la infección periodontal, sus factores de virulencia para entender la respuesta del huésped y de esta manera establecer estrategias preventivas y terapéuticas particulares a cada caso. Es importante que junto a la inspección clínica y diagnóstico radiográfico, se indiquen exámenes microbiológicos de los individuos con periodontitis, debido a que mediante ellos se podrá determinar la presencia de un tipo específico de microorganismo así como establecer la sensibilidad ante los antibióticos a utilizar como terapia coadyuvante del tratamiento periodontal.

\section{Referencias bibliográficas}

I. Page RC, Kornman KS. The pathogenesis of human periodontitis: an introduction. Periodontology 2000 1997; 14: 9 - II.

2. Hojo K., Nagaoka S., Ohshima T., Maeda N. Bacterial Interactions in Dental Biofilm Development. J. Dent. Res. 2009; 88(II):982990.

3. Liébana J., Castillo A, Alvarez M. Enfermedades periodontales: consideraciones microbiológicas. Med. Oral Cir. Bucal 2004, 9 Supl.: 75 -9l

4. Escudero-Castaño N., Perea-García MA, Bascones-Martínez A. Revisión de la periodontitis crónica: Evolución y su aplicación clínica. Av Periodon Implantol. 2008, 20 (I): 27 - 37.

5. Socransky S, Haffajee A. Microbiología de la enfermedad periodontal. Capitulo 4. En: Lindhe J. Periodontología Clínica e Implantología odon- tológica. 2005.

6. Rupani D., Izano E, Schreiner H, Fine D, Kaplan J. Aggregatibacter actinomycetemcomitans serotype $\mathrm{f}$ O-polysaccharide mediates coaggregation with Fusobacterium nucleatum. Oral Microbiol Immunol 2008; 23 (2): 127 - 130

7. Zambón JJ, Christersson LA, Slots J. Actinobacillus actinomycetemcomitans in human periodontal disease. Prevalence in patient groups and distribution of biotypes and serotypes within families. J. Periodontol 1983; 54 (I2): 707 - II.

8. Fujise O., Wang Y, Chen W, Chen C. Adherence of Aggregatibacter actinomycetemcomitans vía serotype-specific polysaccharide antigens in lipopolysaccharides.Oral Microbiol. Inmunol 2008; 23: 226 - 233.

9. Gutiérrez-Venegas G., Contreras-Marmolejo L, Román-Alvárez P, Barajas-Torres C. Aggregatibacter actinomycetemcomitans lipopolysaccharide affects human gingival fibroblast cytoskeletal organization. Cell Biol Int. 2008; 32 (4): 417 - 426.

10. Takada K., Saito M, Tsuzukibashi O, Kawashima Y, Ishida S, Hirasawa M. Characterization of a new serotype $g$ isolate of Aggregatibacter actinomycetemcomitans. Molecular Oral Microbiol 2010; 25 (3): 200 - 206.

II. Kawamoto D., Ando ES, Longo P. L., Nunes AC, Wikström M, Mayer M. Genetic diversity and toxic activity of Aggregatibacter actinomycetemcomitans isolates. Oral Microbiol Immunol 2009; 24: 493 - 50 I.

12. Paton C. Actinobacillus actinomycetemcomitans: su susceptibilidad a los microbianos. Tesis doctoral. Universitat de Barcelona. 2007. www. tdr.cesca.es/TESIS_UB/AVAILABLE/TDX../CEP_ TESIS.pdf

13. Asikainen, S., Lai $\mathrm{CH}$, Alaluusua S, Slots J. Distribution of Actinobacillus actinomycetemcomitans serotypes in periodontal health and disease. Oral Microbiol Immunol 1991; 6: 115 $-118$.

14. Chen C., Wang T., Chen W. Occurrence of Aggregatibacter actinomycetemcomitans serotypes in subgingival plaque from United States subjects. Molecular Oral Microbiol 2010; 25 (3): 207 - 214.

15. Holbrook W.P., Mooney J, Sigurdson T, Kitsiu N, Kinane D. Putative periodontal pathogens, antibody titres and avidities to them in a longitudinal studys patients with resistant periodontitis. J Clin Periodontol I98I; 8 (3): I55 - 164.

16. Holtta P, Alaluusua S, Saarela M, Asikainen S. Isolation frequency and serotype distribution of mutans streptococci and Actinobacillus actinomy- 
cetemcomitans, and clinical periodontal status in Finnish and Vietnamise children Scand J Den Res 1994; 102 (2): II3 - II9.

17. Roman-Torres, C. Prevalence and distribution of serotype-specific genotypes of Aggregatibacter actinomycetemcomitans in chronic periodontitis Brazilian subjects. Ar Oral Biol 2010; 55 (3): 242 - 248.

18. Lopez Pinedo, M. Actinobacillus actinomycetemcomitans y Porphyromona gingivalis en relación a las periodontitis agresivas. Rev. Estomatol. Herediana 2005; 15 (2): 178 - 183.

19. Lima F. L., Roque de Carvalho M, Morais Apolônio A, Porto Bemquerer M, Matos Santoro $M$, Silvano Oliveira et al. Actinomycetemcomitin: a new bacteriocin produced by Aggregatibacter (Actinobacillus) actinomycetemcomitans. J Ind Microbiol Biotechnol 2008; 35: 103 - II0.

20. Olsen I, Shah H, Gharbia S. Taxonomv and biochemical characteristics of Actinobacillus actinomycetemcomitans and Porphyromonas gingivalis Periodontol 2000 1999; 20: 14 - 52.

21. Gallant C., Sedic M, Chicoine E, Ruiz, Mintz K. Membrane morphology and leukotoxin secretion are associated with a novel membrane protein of Aggregatibacter actinomycetemcomitans. J Bacteriol 2008; 90 (17): 5972 - 5980.

22. Potempa, J., Banbula A, Travis J. Role of bacterial proteinases in matrix destruction and modulation of host responses. Periodontol 20002000 Oct; 24: 153 - 192.

23. Kiili, M., Cox SW, Chen HW, Wahlgren J, Maisi P, Eley BM, Salo T and Sorsa T. Collagenase-2 (MMP-8) and collagenase-3 (MMP-I3) in adult periodontitis: molecular forms and levels in gingival crevicular fluid and immunolocalisation in gingival tissue. J Clin Periodontol 2002, 29: 224 $-232$.

24. Queiroz A, Taba M, O' Connell $P$ et al. Inflammation markers in healthy and periodontitis patients. A preliminary data screening. Braz Dent J 2008; 19 (I): 3 - 8

25. Kinane D., Mooney J, Ebersole J. Humoral immune response to Actinobacillus actinomycetemcomitans and Porphyromonas gingivalis in periodontal disease. Periodontology 2000 2000; 20: 289 - 340.

26. Rams T., Listgarten M, Slots J. Actinobacillus actinomycetemcomitans and Porphyromonas gingivalis subgingival presence, species-specific serum immunoglobulin $\mathrm{G}$ antibody levels, and periodontitis disease recurrence. J. Periodont. Res 2006; 4I: 228 - 234.

27. Frías Lopez, M., Herrera Ureña J, Carasol Campillo M, Donate Castro E. Diagnóstico de la enfermedad periodontal basado en la respuesta del huésped. Cient Dent. 2007; 4 (2): 159 - 169.

28. Slots J. Selective médium for isolation of Actinobacillus actinomycetemcomitans. J Clin Microbiol 1982; 15 (4): 606 - 609.

29. Tsuzukibashi Takada O, Saito M, Kimura C, Yoshikawa T, Makimura M., Mirasawa M. A novel selective medium for isolation of Aggregatibacter (Actinobacillus) actinomycetemcomitans. J. Periodontol. Res. 2008; 43: 544 - 548.

30. Bonta $Y$, Zambón J, Genco R, Neiders $M$. Rapid Identification of Periodontal Pathogens in Subgingival Plaque: Comparison of Indirect Immunofluorescence Microscopy with Bacterial Culture for Detection of Actinobacillus actinomycetemcomitans. J Dent Res 1985; 64(5):793-798.

31. Van Vinkelhof AJ, Rams TE, Slots J. Sitemic antibiotic therapy in periodontics. Periodontol 2000 1996; 10: 45- 78

32. 32.- Gmur R, Guggenheim B. Interdental supragingival plaque a natural habitat of Actinobacillus actinomycetemcomitans, Bacteroides forsythus, Campilobacter rectus and Prevotella nigrescens. J Dent Res. 1994; 73: I42I - 1428

33. Bascones A., Caballero A. Actinobacillus actinomycetemcomitans y Porphyromonas gingivalis como principales patógenos periodontales. Avances en Periodoncia 2000 sep; 12 (2): 69 - 75

34. Discepoli N, Bascones-Martínez A. Controversias etiológicas, diagnósticas y terapéuticas de la periodontitis agresiva. Av Periodon Implantol 2008; 20 (I): 39 - 47.

35. Mombelli A., Casagni F, Madianos P. Can presence or absence of periodontal pathogens distinguish between subjects with chronic and aggressive periodontitis? A systematic review. J Clin Periodontol 2002; 29 (3): 10 - 21.

36. Wang D., Kawashima Y, Nagasawa T, Takeuchi Y, Kojima T, Umeda M et al. Elevated serum IgG titer and avidity to Actinobacillus actinomycetemcomitans serotype $\mathrm{c}$ in Japanese periodontitis patients. Oral Microbiol. Immunol 2005; 20 (3): 172 - 179.

37. Ehmke B, Moter A, Beikler T, Milian E, Flemmig T. Adjunctive antimicrobial therapy of periodontitis: long -term effects on disease progression and oral colonization J Periodontol 2005; 76: 749 - 759.

38. Acuña A., Hernández M. Uso de Antibióticos en el Tratamiento de la Periodontitis Crónica: Revisión de la Literatura. Rev Chil Periodon Oseoint 2006, 3 (3): 14 - 22

39. Ardila Medina CM. Eficacia de la moxifloxacina en infecciones odontogénicas. Av Odontoestomatol 2009; 25 (4): 215 - 222. 